\title{
Colorectal goblet cell mucins in familial adenomatous polyposis
}

\author{
K SUGIHARA, ${ }^{*} \dagger$ J R JASS* $\ddagger$
}

From the *Imperial Cancer Research Fund, Colorectal Cancer Unit, St Mark's Hospital, London, the $\dagger$ Department of Surgery, University of Tokyo, Japan, and the $\ddagger$ Department of Histopathology, Medical College of St Bartholomew's Hospital, London

SUMMARY Ninety two tissue blocks from the left colon and 52 from the right colon were obtained from 112 patients with familial adenomatous polyposis (FAP). Tissues from 137 patients with other conditions served as controls. Within the main study a smaller investigation was performed to compare sections from the left and right colon in the same subject. Several well known histochemical techniques were used to investigate possible changes in sulphation, sialic acid structure (loss of $\mathrm{O}$-acetyl substituents), and changes in the ratio of sialic acid to neutral sugars. In patients with FAP, as in controls, there was increased expression of periodic acid Schiff positive mucin and fucose in the right colon. The only difference between patients with FAP and controls was the indirect demonstration of less neutral mucin in the right colon in FAP, but this did not seem to affect neutral sugars binding to UEA-1, PNL, or HPA. As in the general population, a small proportion of patients with FAP showed a lack of O-acetyl substituted sialic acid. Sialic acid heterogeneity probably has a genetic basis, but this is not associated with the genetic defect underlying FAP.

Several histochemical studies have shown associations between carcinogenesis and modifications to goblet cell mucin in the large intestine. Filipe proposed that increased secretion of sialomucin could indicate an early stage of carcinogenesis in the large intestine. ${ }^{1}$ Culling and Reid suggested that reduced $\mathrm{O}$-acetylation of the hydroxy side chain of sialic acid was a prominent feature of both large bowel cancer and adjacent normal mucosa. ${ }^{2}{ }^{3}$ Unfortunately, these studies lacked tissue controls. Furthermore, the histochemical techniques used in these studies have been criticised for their non-specificity. Using mild periodic acid Schiff (mPAS) and PAS modifications, we have shown that there is no difference between normal mucosa adjacent to cancer and control tissues in terms of any reduced expression of $\mathrm{O}$-acetyl sialic acid. ${ }^{4}$ We also confirmed the existence of important regional differences in the large intestine in relation to the expression of sialic acid and neutral mucin. ${ }^{4}$

Several investigations ${ }^{5-7}$ have reported mucin changes in apparently normal goblet cells in patients with familial adenomatous polyposis (FAP). Such observations might be important in providing insight

Accepted for publication 9 December 1986 into the mechanism of carcinogenesis and could turn out to be useful techniques for early diagnosis. These studies, however, have been limited by the number of cases, failure to heed regional differences of goblet cell mucin, a lack of control tissues, and the use of staining methods of doubtful specificity.

We have now examined a large number of colectomy specimens from patients with FAP, using histochemical techniques of acceptable specificity to uncover any mucin changes in apparently normal goblet cells.

\section{Material and methods}

Paraffin embedded tissue blocks taken from 112 colectomy specimens, which had been removed because of FAP, were obtained from the archives of the pathology department at St Mark's Hospital. Ninety two blocks originating from the left colon and 52 from the right colon were selected. Normal mucosal samples from 32 patients with diverticular disease, 32 with irritable bowel syndrome, and 25 with chronic constipation were used as controls for the left colon. Twenty three patients with chronic constipation and 25 with Crohn's disease served as controls for the right colon. Twenty five patients with FAP 
were matched with patients with chronic constipation by age and sex. In 22 of these matched cases specimens from both the left and right colon were available; this provided an important opportunity to assess regional differences of mucin expression in individual patients.

All specimens were fixed in $10 \%$ neutral buffered formalin, routinely embedded, serially sectioned at a thickness of $5 \mu \mathrm{m}$, and stained by haematoxylin and eosin and the following histochemical methods:

1 Mild sodium periodate oxidation/Schiff (mPAS) ${ }^{8}$ to show sialic acid lacking O-acetyl groups. Neutral mucin is not stained.

2 Saponification/periodic acid/phenylhydrazine/Schiff (KOH/PAPS). Removal of ester on the hydroxy side chain by saponification ${ }^{9}$ renders all forms of sialic acid oxidisable with periodic acid. Neutral mucin is blocked with phenylhydrazine. ${ }^{10}$

$3 \mathrm{High}$ iron diamine/alcian blue (HID/AB). ${ }^{11}$ A modification of the method of Spicer was used, using $60 \% \mathrm{FeCl}_{3}$. Diamine reagents were obtained from Kodak Ltd. This method distinguishes sulphated acid mucins (brown stain) and nonsulphated acid mucins (blue stain).

4 Periodic acid/Schiff (PAS). Stains neutral mucin and sialomucin lacking $\mathrm{O}$-acetyl groups.

5 Periodic acid/phenylhydrazine/Schiff (PAPS). ${ }^{10-13}$ Phenylhydrazine blocks periodate reactivity due to neutral mucin. The results are similar to those obtained with mPAS. ${ }^{4}$

6 Tissue binding patterns with three lectins were visualised with the following direct immunoperoxidase technique (table 1).

i Rehydration.

ii Incubate at $37^{\circ} \mathrm{C}$ in $0.1 \%$ trypsin in $0.1 \%$ $\mathrm{CaCl}_{2}$ (pH 7.8) for 20 minutes.

iii Wash in PBS for five minutes.

iv Block endogenous peroxidase with $3 \%$ hydrogen peroxide for five minutes.

$v$ Wash in PBS for five minutes.

vi Incubate in $50 \mu \mathrm{g} / \mathrm{ml}$ horseradish peroxidase conjugated lectin for 30 minutes at room temperature.

vii Wash three times in PBS for 15 minutes.

viii Develop peroxidase with 3,3'-diamino-

Table 1 Lectins and their specificities

\begin{tabular}{lll}
\hline Lectin & $\begin{array}{l}\text { Major sugar binding } \\
\text { specificity }\end{array}$ & Source \\
\hline $\begin{array}{l}\text { Ulex europeus-I }{ }^{14} \\
\text { Peanut agglutinin }\end{array}$ & $\begin{array}{l}\text { L Fucose } \\
\text { Galactose }^{1} \vec{\rightarrow}^{3} \\
\text { N-Acetylgalactosamine } \\
\text { Galactose } \rightarrow^{3 / 4} \\
\text { N-Acetylglucosamine }\end{array}$ & $\begin{array}{l}\text { E-Y Laboratories } \\
\text { Sigma }\end{array}$ \\
$\begin{array}{l}\text { Helix pomatia } \\
\text { agglutinin }\end{array}$ & N-Acetylgalactosamine & E-Y Laboratories \\
\hline
\end{tabular}

benzidine in Tris buffer ( $\mathrm{pH} 7 \cdot 6$ ) with 4 drops of $0.3 \%$ hydrogen peroxide for 10 minutes.

ix Wash in tap water.

$x$ Dehydrate, clear, and mount.

Known positive and negative cases were included in each staining run as controls. Test sections and matched controls were stained at the same time in procedures 2, 3, 4, 5 and 6 . Statistical evaluation was achieved by the $\chi^{2}$ test and the Wilcoxon rank sum test.

\section{Results}

MILD PAS STAINING

One hundred and forty four samples of normal flat mucosa between adenomas of patients with FAP and 137 normal mucosa samples from control groups were stained with mPAS. The left and right colon were studied separately to eliminate the influence of regional differences in colonic mucin expression. Mild PAS shows two possible staining patterns-diffuse and focal. Diffuse staining was graded according to the intensity as negative $(-)$, weak $(+)$, and intense $(++)$. Focal staining was recorded when isolated crypts stained intensely. There were no differences between FAP and controls in both the left and right colon, in terms of either diffuse or focal staining (table 2).

TOTAL SIALIC ACID, SHOWN WITH KOH/PAPS AND SULPHATED $V$ NON-SULPHATED ACID MUCIN (SIALOMUCIN) SHOWN WITH HID/AB Left colonic mucosa samples from 25 patients with FAP and 25 with chronic constipation (matched by age and sex) were used in this study. KOH/PAPS stains all forms of sialic acid. This staining pattern was graded into four groups according to the intensity of staining $(-,+,++,+++)$. HID/AB staining was divided into three patterns; brown predominant, blue predominant, and mixed. With both $\mathrm{KOH} / \mathrm{PAPS}$ and HID/AB staining, there was no noticeable difference between FAP and control cases (tables 3 and 4). Of 39 brown predominant cases with $\mathrm{HID} / \mathrm{AB},+++$ positivity with $\mathrm{KOH} / \mathrm{PAPS}$ was

Table 2 Diffuse staining with mild PAS in patients with FAP and in controls

\begin{tabular}{|c|c|c|c|c|}
\hline & \multicolumn{2}{|c|}{ Left colon } & \multicolumn{2}{|c|}{ Right colon } \\
\hline & $F A P$ & Control & $F A P$ & Control \\
\hline \multirow[t]{2}{*}{$\begin{array}{l}- \\
+ \\
++\end{array}$} & $\begin{array}{l}36 \\
46 \\
10\end{array}$ & $\begin{array}{r}47 \\
34 \\
8\end{array}$ & $\begin{array}{r}28 \\
15 \\
9\end{array}$ & $\begin{array}{r}34 \\
11 \\
3\end{array}$ \\
\hline & 92 & 89 & 52 & 48 \\
\hline
\end{tabular}


Table $3 \mathrm{KOH} / \mathrm{PAPS}$ staining in left colon of 25 patients with FAP and in controls

\begin{tabular}{lcc}
\hline & FAP & Control \\
\hline- & 0 & 0 \\
+ & 1 & 3 \\
++ & 12 & 13 \\
+++ & 12 & 9 \\
& 25 & 25 \\
\hline
\end{tabular}

Table $4 H I D / A B$ staining in left colon of 25 patients with $F A P$ and in controls

\begin{tabular}{lcc}
\hline & FAP & Control \\
\hline Brown predominant & 19 & 20 \\
Mixed & 5 & 3 \\
Blue predominant & 1 & 2 \\
& 25 & 25 \\
\hline
\end{tabular}

observed in $17,++$ positivity in 20 , and + positivity in two. On the other hand, of three blue predominant cases, two were graded ++ , and the other was graded + . From these observations, it is clear that a brown reaction product does not indicate the absence of sialomucin, but simply the presence of sulphate. Conversely, a blue result may not necessarily indicate an abundance of sialomucin but could be due to sulphate that is failing to stain with HID.

\section{EFFECT OF PHENYLHYDRAZINE BLOCKADE ON PAS REACTIVITY}

Both left and right colon samples from 22 patients with FAP and 22 with chronic constipation (matched by age and sex) were stained with PAS and PAPS to identify neutral mucin. The amount of neutral mucin was estimated by assessing the reduction in PAS staining after phenylhydrazine blockade. The effect of phenylhydrazine blockade was graded: no or slight reduction (in PAS staining) $(-)$, moderate $(+)$, and pronounced $(++)$. In the left colon there was no difference between FAP and controls, but in the right colon a significant difference was observed $(p<0.01)$, indicating reduced amount of neutral mucin in the

Table 5 Effect of phenylhydrazine interposition on PAS staining

\begin{tabular}{|c|c|c|c|c|}
\hline & \multicolumn{2}{|c|}{ Left colon } & \multicolumn{2}{|c|}{ Right colon } \\
\hline & $F A P^{*}$ & Controlt & $F A P^{*} \ddagger$ & Control $\ddagger$ \\
\hline \multirow[t]{2}{*}{$\begin{array}{l}- \\
+ \\
+\end{array}$} & $\begin{array}{r}18 \\
4 \\
0\end{array}$ & $\begin{array}{r}12 \\
10 \\
0\end{array}$ & $\begin{array}{r}7 \\
12 \\
3\end{array}$ & $\begin{array}{r}2 \\
11 \\
9\end{array}$ \\
\hline & 22 & 22 & 22 & 22 \\
\hline
\end{tabular}

${ }^{*} p<0.05 ; \mathrm{p} p<0.005 ; \mathrm{fp}<0.01$.
Table 6 UEA-I binding of 22 patients with FAP and in controls

\begin{tabular}{|c|c|c|c|c|}
\hline & \multicolumn{2}{|c|}{ Left colon } & \multicolumn{2}{|c|}{ Right colon } \\
\hline & $F A P^{*}$ & Controlt & $F A P^{*}$ & Controlt \\
\hline \multirow[t]{2}{*}{$\begin{array}{l}- \\
+ \\
+\end{array}$} & $\begin{array}{r}12 \\
2 \\
8\end{array}$ & $\begin{array}{r}11 \\
6 \\
5\end{array}$ & $\begin{array}{r}1 \\
1 \\
20\end{array}$ & $\begin{array}{r}0 \\
3 \\
19\end{array}$ \\
\hline & 22 & 22 & 22 & 22 \\
\hline
\end{tabular}

${ }^{*} \mathrm{p}<0.001 ; \uparrow \mathrm{p}<0.001$.

right colon of patients with FAP (table 5). Controls were also shown to have more neutral mucin in the right colon than the left $(p<0.005)$.

\section{LECTIN STAINING}

The same specimens as those in the preceding study were used. Staining results were classified into three groups according to the numbers of goblet cells stained by a lectin: - indicates no staining, + up to $50 \%$, and ++ more than $50 \%$. When goblet cells showed no Ulex europeus-I binding, columnar cells were sometimes stained intensely, particularly in the crypt base. There was no difference between FAP and controls in both the left and right colon (table 6). The right colon, however, was found to have more $\alpha-\mathrm{L}$ fucose than the left colon in both FAP and control tissues $(p<0.001)$. In both FAP and controls no goblet cell mucin was stained with peanut agglutinin while in some cases the supranuclear region of columnar cells and possibly goblet cells showed peanut agglutinin binding. More than $80 \%$ of specimens showed Helix pomatia agglutinin binding, but there was no statistical difference between patients with FAP and controls.

\section{Discussion}

Large bowel mucosa from patients with familial adenomatous polyposis provides an excellent model for investigating functional changes that might precede overt neoplastic change. Although there have been a number of publications describing histochemical changes in normal flat colonic mucosa from patients with FAP, the findings should be interpreted with caution. Filipe found the abnormal staining patterns for HID/AB in histologically normal flat mucosa in FAP $^{5}$ and has used these data to support her hypothesis ${ }^{1}$ implicating the increased expression of non-sulphated sialomucin as an early step in large bowel carcinogenesis. Our demonstration of a discordance between staining by the HID/AB and $\mathrm{KOH} / \mathrm{PAPS}$ techniques, however, would support McFadden's view ${ }^{15}$ that sialomucin is underestimated by the HID/AB method. HID/AB cannot be used to quantitate goblet cell sialomucin. Further- 
more, we found no differences between FAP and con- in this study.

trols using this technique.

A blue colour with the PAT/KOH/PAS technique ${ }^{2}$ has been stated to show decreased O-acetylation of the hydroxy side chain of sialic acid, and has again been interpreted as a premalignant change. ${ }^{3}$ Using the same histochemical technique Muto et al $^{7}$ showed a higher incidence of blue staining in the normal flat mucosa of FAP than in controls. This modified PAS technique is not specific to sialic acid, however; a blue colour may also be due to neutral mucin. In the study of Muto et al the left and right colon were not identified and studied separately. Mild PAS, developed by Veh, ${ }^{8}$ provides a very simple method to show sialic acid lacking $\mathrm{O}$-acetyl groups. An intense mPAS result, showing sialic acid lacking O-acetyl groups, occurred with the same frequency in FAP patients as in controls. We have previously shown that an intensely positive reaction with mPAS (affecting the entire goblet cell population of the large intestine) is found in about $9 \%$ of all subjects. ${ }^{4}$ We suggested that this sialomucin heterogeneity has a genetic basis, mediated through the expression of $\mathrm{O}$-acetyl transferase. Our findings, however, exclude any association between this gene and the gene defect underlying FAP.

UEA-I binds specifically to terminal $\alpha$-L fucose of the glycoprotein macromolecule. ${ }^{14}$ Yonezawa et al reported that all of four cases with FAP had UEA-I binding receptors in the normal flat mucosa of the left colon, ${ }^{6}$ whereas most goblet cell mucins of 29 normal left colonic mucosae were not bound. ${ }^{17}$ In our study no difference in UEA-I binding was found between those with FAP and controls in either the left or right colon, but a pronounced regional difference in both those with FAP and controls (more fucose in the right colon) was confirmed. Biochemical investigations by Reid et $a l^{18}$ have also shown a greater expression of fucose in the right than left colon.

We showed a reduction in neutral mucin in the right colon of patients with FAP as compared with that of controls; this might reflect a loss of neutral sugars other than those binding to UEA-I, PNL, and HPA. Further research is required.

Our failure to detect mucin changes in the normal appearing mucosa in patients with FAP contradicts the findings of others, ${ }^{5-7}$ but would fit with Knudson's hypothesis regarding the genetic susceptibility to tumours such as retinoblastoma. ${ }^{19}$ According to this hypothesis, a recessive gene implicated in the control of differentiation is inherited and a second mutation in the homologous gene is required to interrupt synthesis of the gene product. This second mutation occurs sufficiently of ten to make the phenotypic expression a dominant characteristic. Given this hypothesis one would expect adenomas in FAP to begin as focal abnormalities in otherwise normal appearing mucosa. This is precisely what we observed

References

1 Filipe MI, Branfoot AC. Abnormal patterns of mucus secretion in apparently normal mucosa of large intestine with carcinoma. Cancer 1974;34:282-90.

2 Culling CFA, Reid PE, Worth J, Dunn WL. A new histochemical technique of use in the interpretation and diagnosis of adenocarcinoma and villous lesions in the large intestine. $J$ Clin Pathol 1977;30:1056-62.

3 Reid PE, Owen DA. Dunn WL, Ramey CW, Lazosky DA, Clay MG. Clinical and histochemical studies of normal and diseased human gastrointestinal tract. III. Changes in the histochemical and chemical properties of the epithelial glycoproteins in the mucosa close to colonic tumours. Histochem J 1985;17:171-81.

4 Sugihara K, Jass JR. Colorectal goblet cell mucin heterogeneity and its relation to malignant disease. J Clin Pathol 1986;39:1088-95.

5 Filipe MI, Mughal S, Bussey HJ. Patterns of mucus secretion in the colonic epithelium in familial polyposis. Invest Cell Pathol 1980;3:329-43.

6 Yonezawa S, Nakamura T, Tanaka S, Maruta K, Nishi M, Sato E. Binding of Ulex europaeus agglutinin-I in polyposis coli: comparative study with solitary adenoma in the sigmoid colon and rectum. $J N C I$ 1983;71:19-24.

7 Muto T, Kamiya J, Sawada T, Agawa S, Morioka Y, Utsunomiya J. Mucin abnormality of colonic mucosa in patients with familial polyposis coli. Dis Colon Rectum 1985;28:147-8.

8 Veh RW, Meessen D, Kuntz D, May B. In: Malt RA, Williamson RCN, eds. Colonic carcinogenesis. Lancaster: MTP Press Ltd, 1982:355-65.

9 Culling CFA, Reid PE, Dunn WL. The effect of saponification upon certain histochemical reactions of the epithelial mucin of G.I. tract. Histochem Cytochem 1971;19:654-62.

10 Spicer SS. The use of various cationic reagents in histochemical differentiation of mucopolysaccharides. Am J Clin Pathol 1961;5:393-407.

11 Spicer SS. Diamine methods for differentiating mucosubstances histochemically. J Histochem Cytochem 1965;13:211-34.

12 Reid PE, Dunn WL, Ramey CW, Coret E, Trueman L, Clay MG. Histochemical studies of the mechanism of the periodic acidphenylhydrazine-Schiff (PAPS) procedure. Histochem $J$ 1984;16:641-9.

13 Lev R, Lance P, Camara P. Histochemical and morphologic studies of mucosa bordering rectosigmoid carcinomas: comparisons with normal, diseased and malignant colonic epithelium. Hum Pathol 1985;16:151-61.

14 Matsumoto I, Osawa T. Purification and characterization of an anti-H(O) phytohemagglutinin of Ulex Europeus. Biochim Biophys Acta 1969;194:180-9.

15 Picard JK, Feizi T. Peanut lectin and anti-Ii antibodies reveal structural differences among human gastrointestinal glycoproteins. Mol Immunol 1983;20:1215-20.

16 McFadden DE, Owen DA, Reid PE, Jones EA. The histochemical assessment of sulphated and non-sulphated sialomucin in intestinal epithelium. Histopathology 1985;9:1129-37.

17 Yonezawa S, Nakamura T, Tanaka S, Sato E. Glycoconjugate with Ulex europaeus agglutinin-I-binding sites in normal mucosa, adeonoma, and carcinoma of the human large bowel. JNCI 1982;69:777-85.

18 Reid PE, Culling FA, Dunn WL, Ramey CW, Clay MG. Clinical and histochemical studies of normal and diseased human gastrointestinal tract. I. A comparison between histologically normal colon, colonic tumours, ulcerative colitis and diverticular disease. Histochem $J$ 1984;16:235-51.

19 Knudson AG. Mutation and cancer: statistical study of retinoblastoma. Proc Natl Acad Sci USA 1971;68:820-3.

Requests for reprints to: Dr JR Jass, St Mark's Hospital, City Road, London ECIV 2PS, England. 